\title{
An improved gene synthesis method with asymmetric directions of oligonucleotides designed using a simulation program
}

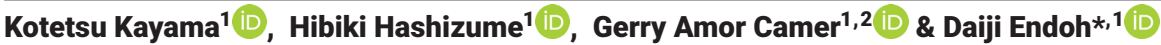

${ }^{1}$ Department of Radiation Biology, School of Veterinary Medicine, Rakuno Gakuen University, 582 Midori-machi Bunkyo-dai, Ebetsu 069-8501, Japan; ${ }^{2}$ College of Veterinary Medicine, University of Eastern Philippines, Catarman, 6400, Northern Samar, Philippines; *Author for correspondence: dendoh@rakuno.ac.jp

BioTechniques 69: 211-219 (September 2020) 10.2144/btn-2020-0062

First draft submitted: 28 April 2020; Accepted for publication: 3 June 2020; Published online: 18 June 2020

\section{ABSTRACT}

Artificial gene synthesis based on oligonucleotide augmentation is known as overlap extension PCR which generates a variety of intermediate synthetic products. The orientation and concentration of oligomers can be adjusted to reduce the synthesis of intermediates and optimize the full-length process of DNA synthesis, using a simulation program for serial oligomer extension. The efficiency of the serial oligomer extension process is predicted to be greatest when oligomers are in a 'forward-reverse-reverse-reverse' direction. Oligomers with such designed directions demonstrated generation of the desired product in the shortest time (number of cycles) by repeated annealing and elongation. This method, named Asymmetric Extension supported by a Simulator for Oligonucleotide Extension (AESOE), has shown efficiency and effectiveness with potentials for future improvements and optimal usage in DNA synthesis.

\section{METHOD SUMMARY}

We developed a cost-effective, user-friendly and reliable protocol with asymmetrically aligned oligonucleotides and a simple thermal cycle for artificial gene synthesis, designed with an oligonucleotide polymerization simulator. This novel protocol is named Asymmetric Extension supported by a Simulator for Oligonucleotide Extension (AESOE).

\section{GRAPHICAL ABSTRACT}

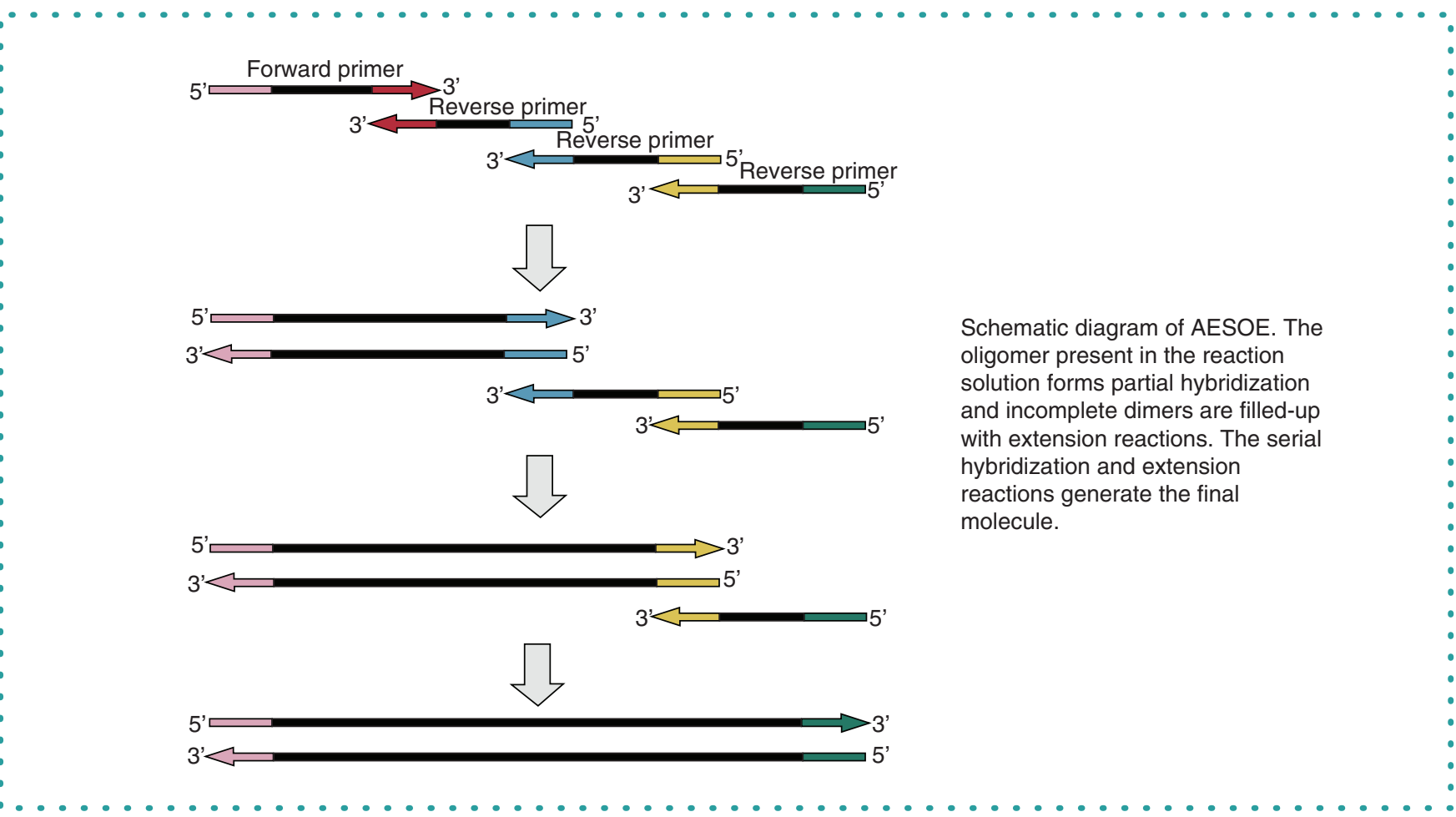




\section{KEYWORDS:}

AESOE • artificial gene synthesis • DNA extension $\bullet$ OE-PCR • partial dimer

Researchers can construct large-scale biological hypotheses that include many gene interactions by analyzing large-scale DNA sequence information [1-8]. To test a biological hypothesis, natural or artificially generated mutant organisms have been used. However, as more efficient methods are developed, construction of DNA as an entity from a designed sequence - for instance, DNA synthesis technology - has begun to be used in engineering and biology [9-11]. DNA reading by next-generation sequencing, combined with DNA synthesis technology, drives synthetic biology efforts and enables detailed predictions regarding biological systems [12-14]. However, the pace of DNA synthesis technology lags behind that of DNA sequencing technology innovations [15]. The former needs to be accelerated at a relatively low cost.

Production of synthetic DNA is commonly referred to as 'gene synthesis.' Specifically, a DNA-length fragment (250-2000 bp) can be synthesized from a chemically manufactured single-stranded synthetic DNA oligonucleotide [16,17]. Chemically synthesized oligonucleotides used for gene synthesis are produced using variations of the phosphoramidite chemistry of either column-based or microarraybased synthesizers [18]. The development of a gene synthesis technology applicable to a microarray-based synthetic oligonucleotide may significantly reduce the cost of gene synthesis.

Early gene synthesis was performed by ligating DNA oligonucleotides that form DNA duplexes without gaps by hydrogen bonding with DNA ligase $[19,20]$. Initially, a continuous ligation reaction has to be performed with additional DNA oligomers, but a published method using thermostable ligase enabled desired reactions in a single tube [21,22].

Following the development of PCR in the 1980s, a ligation-free method was developed using synthetic oligonucleotides that form partially double-stranded hydrogen bonds. Completely synthesized DNAs were then created that require a naturally occurring DNA template [23-25]. Numerous different methods have been created for assembling dsDNA from synthetic oligonucleotides in a PCR-like manner; all these methods use thermostable DNA polymerase and PCR to synthesize dsDNA using synthetic oligonucleotides with complementary overlapping sequences between adjacent oligonucleotides. These methods are called overlap extension PCR (OE-PCR) because DNA synthesis begins as an extension reaction by PCR from the overlapping part [26]. OE-PCR is cost-efficient and generates synthetic products of substantial length [27-31]. It is therefore necessary to determine the base sequence of the overlapping portion by analyzing the dsDNA of the final product [27]. Although OE-PCR is said to be capable of synthesizing dsDNA with a length of more than 1000 bases, in practice, if the total length exceeds 300 nucleotides, OE-PCR requires careful consideration of the composition of the oligomer as well as the linking part of the oligomer.

In this study, we performed and examined an effective method for synthesizing dsDNA by linking chemically synthesized oligonucleotides, akin to OE-PCR. We prepared a program (named as Asymmetric Extension supported by a Simulator for Oligonucleotide Extension, or AESOE) that simulates the process of oligonucleotide synthesis, and which developed a method to efficiently synthesize the final PCR product by adjusting the direction, and by mixing the ratio of the oligonucleotides.

\section{Materials \& methods}

Prediction of reaction by simulation program

We created a simulator on simultaneous hybridizations and polymerase-derived-extensions to design oligomers partially hybridized to each other to generate DNAs of 150-220 bases (Supplementary Table 1). Assuming that extension of DNA fragments occurs by extension of oligomers on partially hybridized oligomers or ssDNAs (Figure 1), an extension of the DNA fragment is dependent on the conformation of the partial dimer against the original duplex. A short oligomer efficiently hybridizes on one strand of the duplex, but when the hybridized region is a part of the oligomer, a partial duplex is made against the full ssDNA (Figure 1, steps 2-4). Because such a reaction is accomplished by proceeding from the terminal oligomer, we predicted that forward-reverse-reverse-reverse (F-R-R-R; left column of Figure 1) would produce the end product in the same way as forward-forward-forward-reverse (F-F-F-R; right column of Figure 1). In this study, the efficient synthesis of oligomers by orientation and composition was verified by using F-R-R-R. The probability of constructing partially hybridized dimers is dependent on three conditions: 1) one of the molecules is single-stranded, 2) the concentration of extending oligomers is high, and 3 ) the extending oligomer is short (Figure 2, left column). Under these conditions, it is assumed that OE-PCR may not be efficient for the construction of partially hybridized ssDNA (Figure 2, right column), because the original duplex is suggested to be more efficient for dimer formation than the partially hybridized dimers (Figure 2, lower right).

\section{DNA fragment sequences for synthesis}

We prepared oligomers to construct parts of Dengue or Zika genes (Table 1). For AESOE reactions, the directions of the oligomers were set for F-R-R-R (4-oligomer construction) or F-R-R (3-oligomer construction) on alignment of the target sequences (Eurofins Genomics Co. Ltd, Tokyo, Japan) (Table 2). Since the overlapping length strongly influences the cost-effectiveness of artificial gene synthesis, overlapping sequences were set at 17 bases, which was suggested to be the minimum requirement for AESOE on preliminary experiments. 


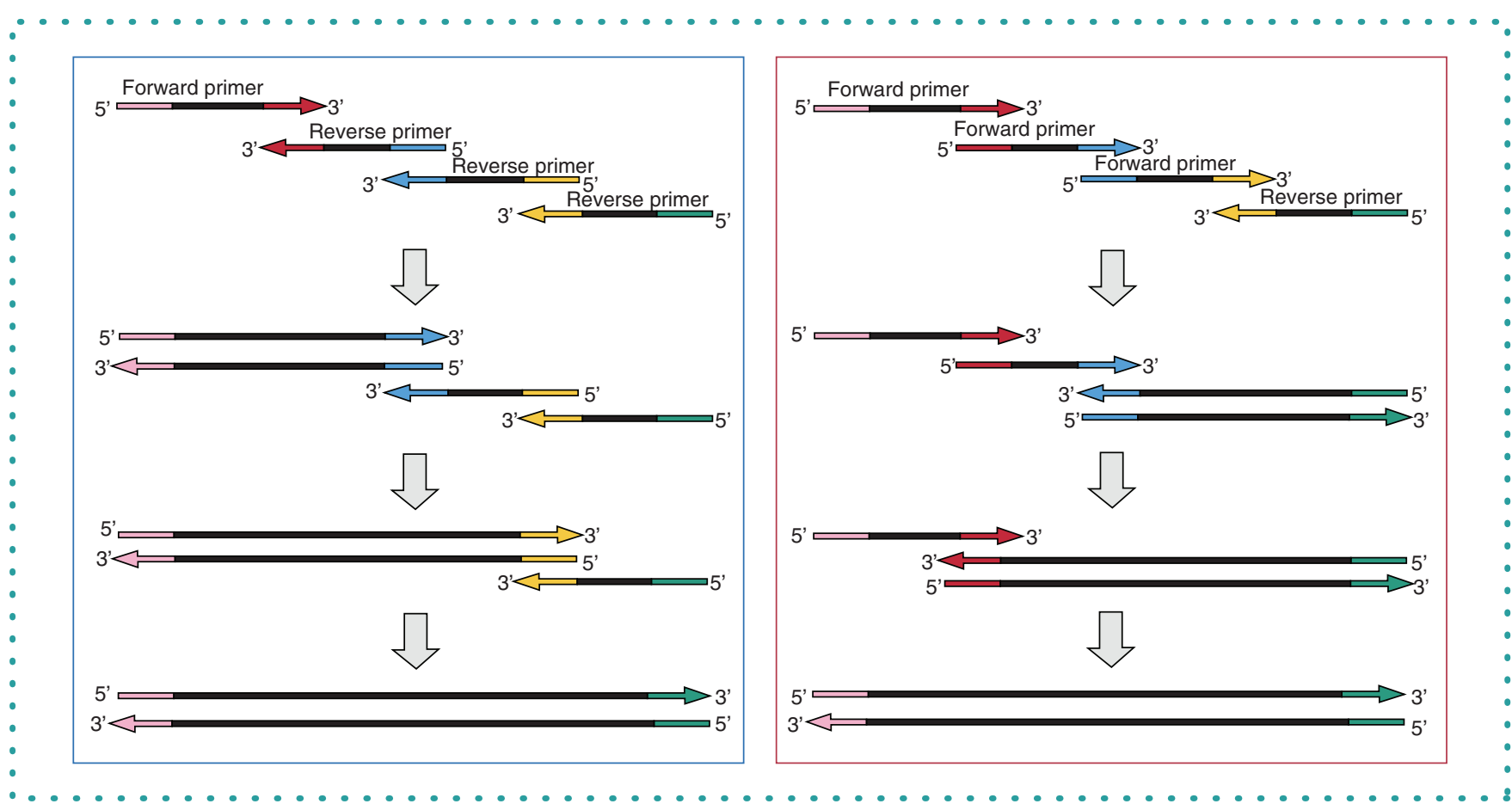

Figure 1. Schematic diagram of the Asymmetric Extension supported by a Simulator for Oligonucleotide Extension reaction. The direction of the arrow indicates the direction of the base sequence of the oligomer with respect to the final product. The colors of the arrowheads and the other end indicate the complementarity of the linked sequences. The left column shows F1-R1-R2-R3 construction. By setting the concentration and adjusting the direction of the oligomer, the synthetic product shows that dsDNA extends in order from the F1 oligomer as shown at the left end. In the same manner, using an F3-F2-F1-R1 construction, dsDNA extends in order from the R1 oligomer (right column).

\section{Hybridization \& extension reactions for AESOE \& agarose gel electrophoresis}

Oligomers were mixed with the ratio: F1:R1:R2:R3 $=25: 1: 5: 25$ or F1:R1:R2 = 25:1:25 to produce an oligomer stock solution. Specifically, $1 \mu \mathrm{l}$ each of $50 \mu \mathrm{M} \mathrm{F1}, 2 \mu \mathrm{M}$ R1, $10 \mu \mathrm{M}$ R2 and $50 \mu \mathrm{M}$ R3 were mixed, and then $275 \mu$ l of distilled water was added to make a four-oligomer stock solution. Similarly, a three-oligomer stock solution was prepared by mixing $1 \mu$ leach of $50 \mu \mathrm{M} \mathrm{F1,2 \mu M} \mathrm{R1} \mathrm{and} 50 \mu \mathrm{M}$ R2, and then adding $276 \mu \mathrm{l}$ distilled water. PCR solution was constructed by mixing $10 \mu$ l of oligomer stock solution with $10 \mu$ GoTaq Green Master Mix (2x; Promega, WI, USA). AESOE reaction conditions were as follows: $95^{\circ} \mathrm{C}$ for 2 min, followed by $2-17$ cycles of $95^{\circ} \mathrm{C}$ for $30 \mathrm{~s}, 30^{\circ} \mathrm{C}$ for $10 \mathrm{~s}$ and $72^{\circ} \mathrm{C}$ for $60 \mathrm{~s}$ (AESOE reaction tube). The AESOE reaction tube was removed from the thermal cycler after $2,4,6,7,8,9,10$, $11,13,15$ or 17 cycles of the reaction. After AESOE reaction, $5 \mu$ of the product was loaded onto a $1.5 \%$ agarose gel containing $1 \times$ TBE running buffer ( $80 \mathrm{mM}$ Tris- $\mathrm{Cl}, 90 \mathrm{mM}$ boric acid and $2 \mathrm{mM}$ EDTA), run at $95 \mathrm{~V}$ for $1 \mathrm{~h}$ and stained with ethidium bromide $(0.5 \mu \mathrm{g} / \mathrm{ml})$.

On the simulator, when the concentration ratio for synthesized duplex and free oligomer was low:high, the extension reaction would be efficient (Supplement 3). Extension was presumed to begin with F1 and R1, making first F1-R1, then F1-R1-R2, and then F1-R1-R2-R3. A concentration ratio F1: R1: R2: R3 = 25:1:5:25 would give a 1:5 ratio of duplex DNA:single-stranded oligomer.

\section{Sequence determination of AESOE products}

dsDNAs synthesized under optimal conditions were sequenced after purification using a PCR purification kit (Gene Elute ${ }^{T M}$ PCR Clean-Up kit, Sigma-Aldrich, MO, USA); sequencing was commissioned to Eurofins Genomics. The nucleotide sequences of all synthetic fragments matched the sequence to be synthesized (data not shown).

\section{Results \& discussion}

\section{Formation of band on agarose gels}

In each lane where the full length of the product was obtained after the cycle, the main band that corresponds to the final product is shown (Supplementary Figure 1). Note that smearing and nonspecific bands were seen prior to obtaining the final main product. Table 2 shows the cycle at which a band matching the product size first appeared in the gel, and the cycle at which this band became the main product. The smearing of the electrophoretic products seen after 2-11 cycles was dependent on the set of oligomer. Fewer oligomers for AESOE resulted in the appearance of a final product in an earlier cycle (Table 2). Assuming that the oligomers and intermediate products are present in double-stranded form, ten products may be produced (Figure $3 \mathrm{~A}$ ). In the AESOE reaction, only three doublestranded fragments are available (Figure 3B). Hybridization of oligomers 1 and 4 resulted in dsDNA (9 \& 10) after elongation. One strand 


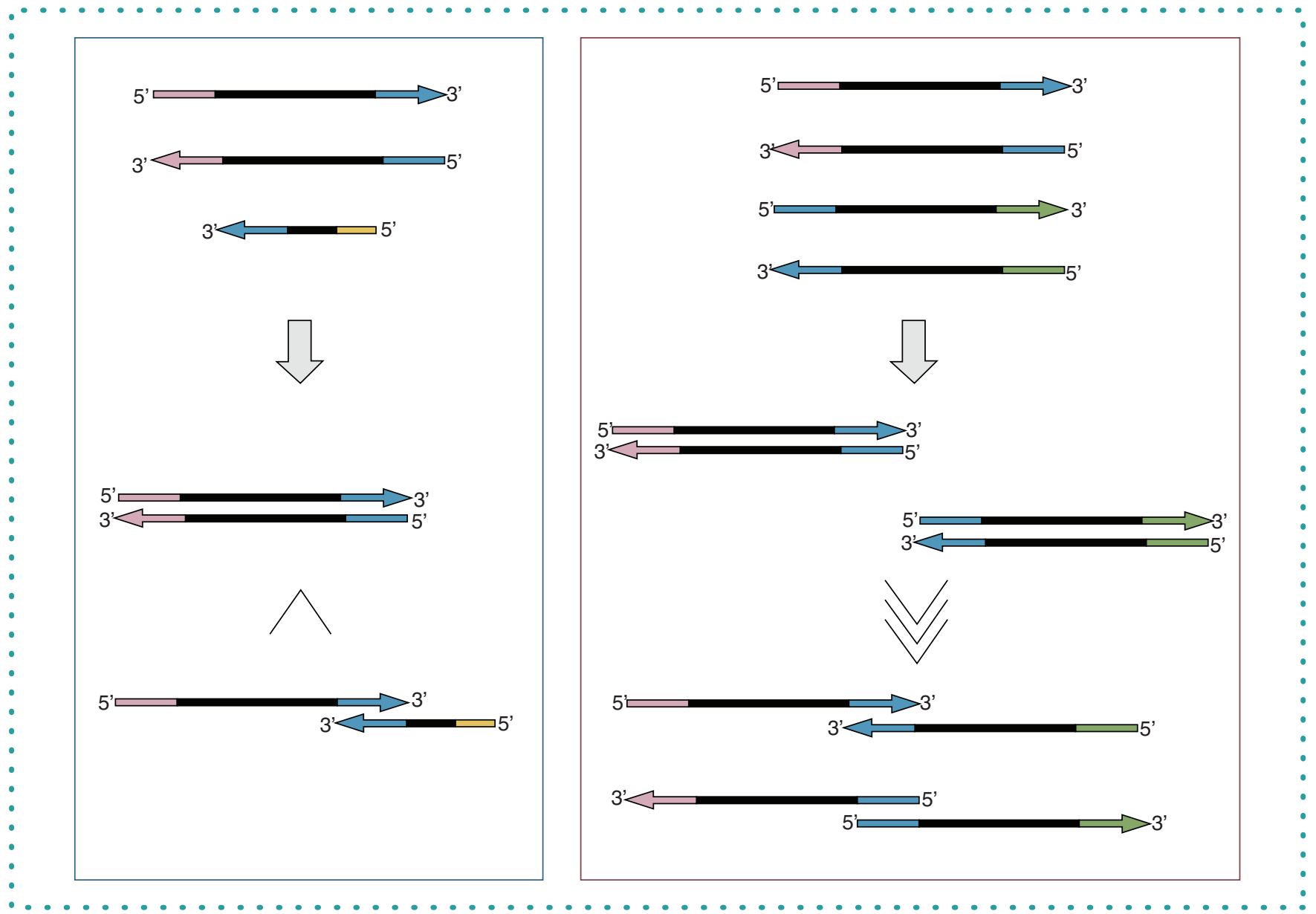

Figure 2. Comparison of conformation efficiency for partial dimers on Asymmetric Extension supported by a Simulator for Oligonucleotide Extension and Overlap Extension PCR. Schematic diagram showing the probability of formation of partial double chains between oligomers. The left column shows annealing when a single-stranded oligomer partially hybridizes with the duplex, and the right column shows annealing when two duplexes form a partial duplex. In this study, we assume a higher probability that a duplex and a partial single-stranded oligomer will optimally hybridize, rather than the probability of two duplexes that will combine to form a partial duplex.

of dsDNA (9) may hybridize with oligomer 6 and result in the next dsDNA (11 \& 12). By similar reaction, all reactions are expected to form the longest dsDNA $(13 \& 14)$. The AESOE theoretically produces the final product in a minimum of three cycles, but the low molarity of oligomers 4 and 6 resulted in 9 and 10 duplexes, and 11 and 12 duplexes in each cycle. Heavy chains are continuously replenished. The band composition after each cycle was consistent with the response models (Supplementary Figure 1).

\section{Sequences of AESOE products}

The nucleotide sequence of the products was determined by Sanger sequencing of the DNAs judged to be the clearest on the gel. The base sequences of AESOE products matched the viral base sequences used for the design (data not shown). These results suggest that the expected fragments were constructed during the elongation reactions. The desired reaction products were produced in fewer than 13 AESOE reaction cycles, suggesting that synthesis errors caused by Taq polymerase could be reduced when compared with those in OE-PCR.

In comparison to the oligomer concentration used in this study, the AESOE product is found to be sufficiently large. By contrast, the number of molecules of PCR product was dependent on the concentration of the end oligomers of the set; the concentrations of F1 and R3 or F1 and R2 (in the four-oligomer and three-oligomer sets, respectively) were suggested as the determinant for the final concentration of AESOE product. The concentration of standard 200-base AESOE products was suggested to be about $10-20 \mathrm{ng} / \mu$ l on the agarose gels compared with the bands of molecular weight markers (Supplementary Figure 1). The mass calculation (10-20) $\times 10^{-9}$ $/(200 \times 660) \times 6.02 \times 10^{23}$ suggested $4.6-9.1 \times 10^{10}$ product molecules per microliter in the loaded product. On the other hand, the molarity of the end-oligomer was $0.09 \mu \mathrm{M}$ in the AESOE reaction solution (see 'Materials \& methods' section), suggesting 0.09 $\times 10^{-6} \times 1 \times 10^{-6} \times 6.02 \times 10^{23}=5.0 \times 10^{10}$ molecules per microliter in the loaded product. These results indicate that a large proportion of oligomers in AESOE reactions were used for the final product after the appropriate number of cycles. It can be inferred that what is 
(A) DNA fragments which may be presented from 4-overlapped short fragments after cycles of denaturation - annealing - extension reaction

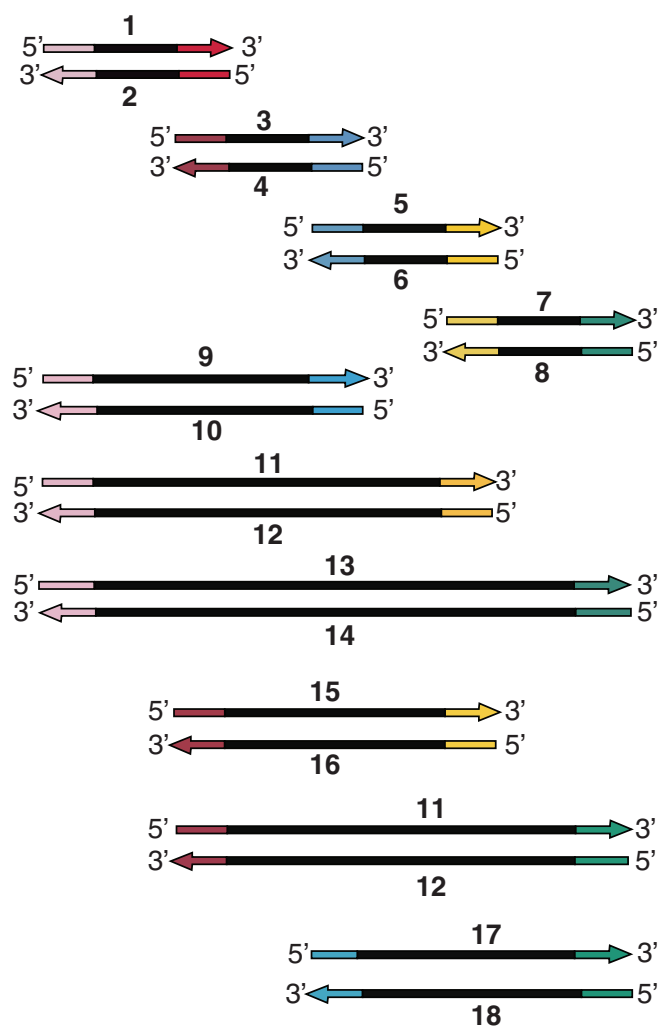

(B) DNA fragments that may be generated in the AESOE reaction

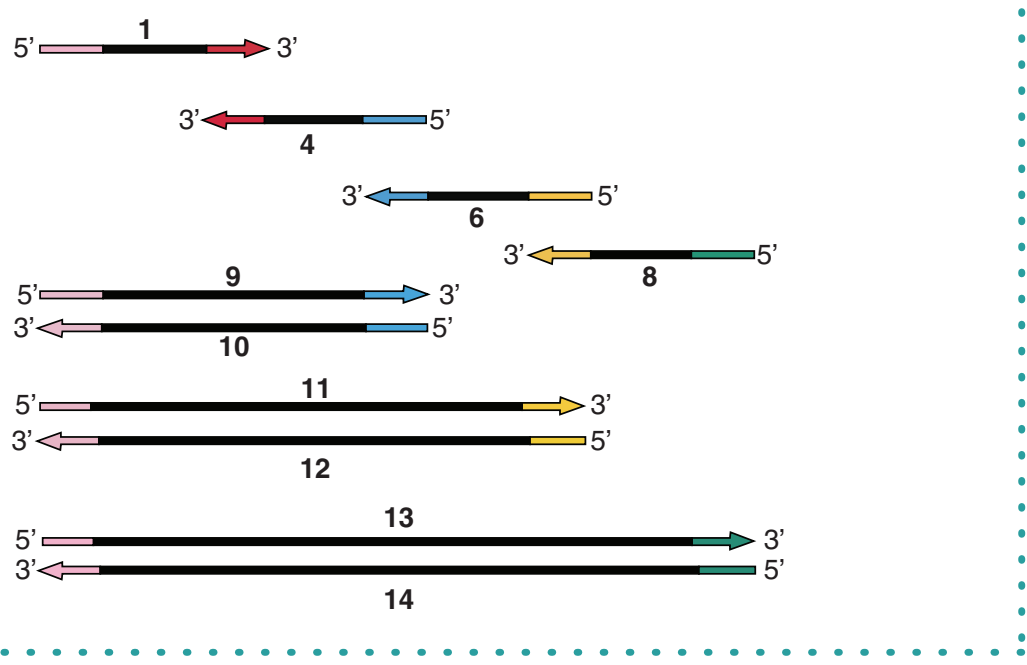

Figure 3. DNA fragments which can be generated during the extension of oligomers. (A) DNA fragments that may be present from four overlapping oligomers after cycles of denaturation/annealing/extension. (B) DNA fragments that may be generated in the AESOE reaction. The types of double-strand DNA fragments suggested are restricted to the model reaction.

AESOE: Asymmetric Extension supported by a Simulator for Oligonucleotide Extension. 


\begin{tabular}{|c|c|c|c|c|c|c|}
\hline Product name & Virus species & Accession & Start position & End position & Fragment length & Number of oligomers \\
\hline DEV1-4-1 & Dengue virus 1 & KY347006.1 & 277 & 485 & 209 & 4 \\
\hline DEV1-4-2 & & KJ189344.1 & 8423 & 8641 & 219 & 4 \\
\hline DEV1-4-3 & & KY347010.1 & 299 & 510 & 212 & 4 \\
\hline DEV2-2-1 & Dengue virus 2 & KF955349.1 & 1871 & 2075 & 205 & 4 \\
\hline DEV2-2-2 & & KF955349.1 & 2059 & 2274 & 216 & 4 \\
\hline DEV2-2-3 & & KF955349.1 & 2258 & 2473 & 216 & 4 \\
\hline DEV2-2-4 & & KF955349.1 & 2457 & 2668 & 212 & 4 \\
\hline DEV2-4-1 & & FJ639705.1 & 7982 & 8190 & 209 & 4 \\
\hline DEV2-4-2 & & FJ639705.1 & 8174 & 8391 & 218 & 4 \\
\hline DEV2-4-3 & & GQ868623.1 & 8375 & 8592 & 218 & 4 \\
\hline DEV2-4-4 & & FJ639705.1 & 8576 & 8793 & 218 & 4 \\
\hline DEV2-4-5 & & FJ639705.1 & 8777 & 8988 & 212 & 4 \\
\hline DEV2-5-1 & & MN018346.1 & 7894 & 8078 & 185 & 4 \\
\hline DEV2-5-2 & & KU517847.1 & 8062 & 8248 & 187 & 4 \\
\hline DEV2-6-1 & & FJ882602.1 & 8557 & 8737 & 181 & 4 \\
\hline DEV2-6-2 & & FJ882602.1 & 8721 & 8912 & 192 & 4 \\
\hline DEV2-6-3 & & FJ882602.1 & 8896 & 9083 & 188 & 4 \\
\hline DEV3-1-1 & Dengue virus 3 & MH544650.1 & 8255 & 8413 & 159 & 3 \\
\hline DEV3-1-2 & & MH544650.1 & 8397 & 8565 & 169 & 3 \\
\hline DEV3-1-3 & & KT726340.1 & 8528 & 8690 & 163 & 3 \\
\hline DEV4-2-1 & Dengue virus 4 & MK506266.1 & 8156 & 8340 & 185 & 4 \\
\hline DEV4-2-2 & & MK506266.1 & 8324 & 8519 & 196 & 4 \\
\hline DEV4-2-3 & & KP406806.1 & 8511 & 8706 & 196 & 4 \\
\hline DEV4-2-4 & & KP406806.1 & 8690 & 8882 & 193 & 4 \\
\hline Zika1 & Zika virus & МT078742.1 & 7802 & 7925 & 124 & 3 \\
\hline
\end{tabular}

important in artificial gene synthesis is not the number of amplifications but the number of generated molecules. If serial elongation of oligomers can directly produce the molecule of interest, then no PCR step involving hybridization of oligomers to the end of a product molecule with subsequent elongation that is repeated 20-to 30-times, is going to be required. At the beginning of the reaction, because the oligomer has a sufficient number of molecules in the solution, the reaction of the oligomer itself is designed in the AESOE so as not to require a PCR reaction to produce a final product. In this study, the target molecule was generated in 4-13 reaction cycles (Supplementary Figure 1, Table 2). As for the length of AESOE products, the number of oligomers and their length may no longer be critical. Therefore the limit of AESOE product is dependent on constructing the desired oligomer length (oligomer length $\times 4-17 \times 3$ ).

Assuming that the primers for PCR reaction are also included in the oligomer, PCR reaction can be viewed as the hybridization between the oligomer and ssDNA along with the resulting DNA extension reaction in an incomplete duplex (Figure 4). In at least one place in OEPCR, the one-sided loops of the two duplexes cross to form an incomplete duplex, whereas in AESOE, all incomplete duplex formation results in at least one oligomer that forms an incomplete duplex without forming a duplex once (Figure 2, right column). The AESOE was designed so that the extension reaction can proceed efficiently, at the same time, increasing the efficiency of hybridization between oligomer and ssDNA on one side of the dsDNA (Figure 2, left column). The short, low-temperature annealing step $\left(30^{\circ} \mathrm{C}, 10 \mathrm{~s}\right)$ is suggested to be suitable for construction of the incomplete dimer. The generation of the target molecule in fewer than 13 cycles in this study is consistent with the hypothesis that incomplete duplexes and extension reactions produce dsDNAs. Comparison of synthesis efficiency between AESOE and OE-PCR suggests that SSDNA of the original duplex and single-stranded oligomers compete with oligomers of the same sense as the oligomers used to synthesize the partial duplex (Supplementary Figure 7). This assumption suggests that the efficiency of partial duplex formation and the formation of new elongated molecules is enhanced when the molar concentration of singlestranded oligomers is greater than that of the original duplex. Considering that annealing after partial dimer formation is a prerequisite for dsDNA synthesis and that partial dimer formation competes with intrinsic double-stranded reconstitution, it is suggested that the orientation and direction of oligomers in AESOE may be a decisive factor in determining the efficiency of gene synthesis.

Engineering hybrid genes without the use of restriction enzymes was first reported in 1989 [26]. In 1997, Urban et al. successfully synthesized 689-base fragments of normal and mutant prokaryote and eukaryote genes by linking oligomers, proposing the name 'overlap extension PCR (OE-PCR)' [27]. In 2003, Gao et al. [28] proposed the 'thermodynamically balanced inside-out' method to adjust the length and overlapping of oligomers, and to synthesize up to 1712 bases by sequential orientation of oligomers. The oligomer orientation method evolved into two-step OE-PCR in 2004, and further improvements in oligomer orientation and the use of high-precision 


\section{Table 2. Cycles in which the band of interest appeared.}

\begin{tabular}{|c|c|c|c|}
\hline \multirow[t]{2}{*}{ Fragment name } & \multirow[t]{2}{*}{ Product size on gel } & \multicolumn{2}{|c|}{ Cycle at which: } \\
\hline & & Final product appeared & Final product became major product \\
\hline DEV1-4-1 & 200 & 8 & 13 \\
\hline DEV1-4-2 & 200 & 9 & 11 \\
\hline DEV1-4-3 & 200 & 8 & 10 \\
\hline DEV2-2-1 & 200 & 7 & 13 \\
\hline DEV2-2-2 & 220 & 8 & 10 \\
\hline DEV2-2-3 & 220 & 7 & 13 \\
\hline DEV2-2-4 & 220 & 7 & 11 \\
\hline DEV2-4-1 & 200 & 7 & 11 \\
\hline DEV2-4-2 & 220 & 7 & 11 \\
\hline DEV2-4-3 & 200 & 7 & 10 \\
\hline DEV2-4-4 & 220 & 7 & 10 \\
\hline DEV2-4-5 & 220 & 6 & 8 \\
\hline DEV2-5-1 & 180 & 6 & 9 \\
\hline DEV2-5-2 & 180 & 6 & 9 \\
\hline DEV2-6-1 & 180 & 6 & 9 \\
\hline DEV2-6-2 & 200 & 7 & 7 \\
\hline DEV2-6-3 & 180 & 8 & 10 \\
\hline DEV3-1-1 & 150 & 4 & 4 \\
\hline DEV3-1-2 & 180 & 4 & 6 \\
\hline DEV3-1-3 & 170 & 4 & 6 \\
\hline DEV4-2-1 & 180 & 10 & 13 \\
\hline DEV4-2-2 & 200 & 6 & 7 \\
\hline DEV4-2-3 & 200 & 6 & 8 \\
\hline DEV4-2-4 & 200 & 10 & 11 \\
\hline Zika1 & 120 & 4 & 4 \\
\hline
\end{tabular}
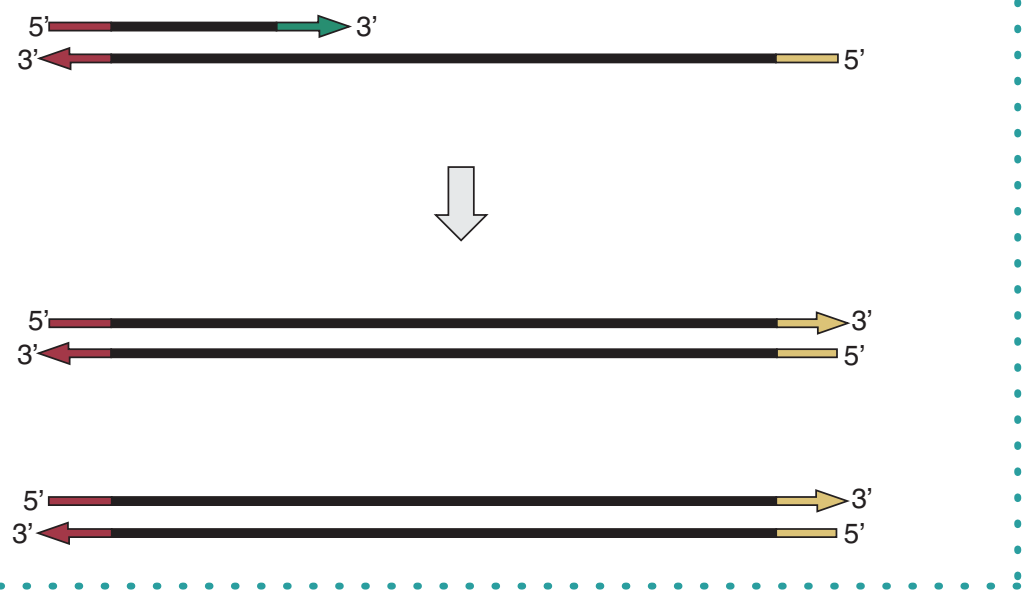

Figure 4. Schematic diagram of a standard PCR reaction. In the PCR reaction, the double-stranded PCR product is reproduced by hydrogen bonding of the primer to one strand on one side of the double-stranded PCR product. This figure shows that, even in the PCR reaction, the formation of incomplete duplexes is the basis for the synthesis of numerous dsDNA fragments. 
polymerases established a technique for synthesizing DNA fragments with sufficient accuracy to exceed the average size of a 2-kb gene [29,30]. In 2014, the addition of design oligomers for an OE-PCR module to FastPCR completed the technique for researchers to synthesize artificial genes [31]. Recently the incorporation of autodegradable peptides has been added to the simultaneous expression of plural proteins [32]. The artificial synthesis of DNA fragments is also being used to prepare standard fragments for PCR design of pathogenic viruses [33]. In the case of standard DNA for viral PCR, the fragment size should be short enough that the gene product has no function, for safety purposes. An improvement made for fast and economical production of 200- to 300-base DNA fragments, multiple overlap extension PCR (MOE-PCR) can also be used to synthesize DNA fragments of more than 600 nucleotides if longer fragments of AESOE DNA are required for research purposes [34-37]. The AESOE method is suggested as an economical, fast and reproducible method for artificial synthesis of DNA fragments that is partially and practically different from OE-PCR gene synthesis.

In conclusion, we have demonstrated that artificial gene synthesis with high production efficiency is made possible by organizing oligomer reactions in the formation of incomplete duplex with subsequent extensions.

\section{Future perspective}

The growing number of emerging pathogens and the threats of the next global pandemic are concerns that could be addressed using advances in synthetic DNA technology. With the relative ease of artificial gene synthesis facilitated by our simple, robust and rapid AESOE method, prospective scientific innovators can now significantly speed up the rapid preparation of DNA fragments needed for synthetic nucleic acid-based therapeutics without the need for complex programs on the design of oligomers. This promising method to readily propagate and characterize genes of interest can promptly progress to respond to the current SARS-CoV-2 pandemic as well as near future pandemics involving other novel pathogens.

\section{Author contributions}

D Endoh and K Kayama conceptualized the study; K Kayama, D Endoh and H Hashizume designed the experiments; K Kayama, D Endoh and GA Camer wrote and critically enhanced the manuscript; $\mathrm{K}$ Kayama and $\mathrm{H}$ Hashizume performed the experiments and prepared tables; K Kayama, H Hashizume and G A Camer prepared the figures; D Endoh analyzed the data, designed a simulator and the source code.

\section{Acknowledgments}

We thank Rakuno Gakuen University, Japan for the funding and Misaki Tanaka for assisting us in PCR-related experiments.

\section{Financial \& competing interests disclosure}

This work was supported by research funding of Rakuno Gakuen University with Lagrange Co. Ltd (Tokyo, Japan). The authors have no other relevant affiliations or financial involvement with any organization or entity with a financial interest in or financial conflict with the subject matter or materials discussed in the manuscript apart from those disclosed. No writing assistance was utilized in the production of this manuscript.

\section{Data sharing statement}

We have shared the computer program in Supplement 3, which is available at https://github.com/daijiendoh/aesoe_extension_simulator

\section{Supplementary data}

To view the supplementary data that accompany this paper please visit the journal website at: www.futurescience.com/doi/suppl/10.2144/btn-2020-0062

\section{Open access}

This work is licensed under the Attribution-NonCommercial-NoDerivatives 4.0 Unported License. To view a copy of this license, visit http://creativecommons.org/licenses/by-nc-nd/4.0/

\section{References}

Papers of special note have been highlighted as: $\bullet \bullet$ of considerable interest

1. Schatz MC, Phillippy AM. The rise of a digital immune system. Gigascience 1(1), 4 (2012).

2. Sayers EW, Agarwala R, Bolton EE et al. Database resources of the National Center for Biotechnology Information. Nucleic Acids Res. 47, D23 (2019).

-• Describes the value of generating huge sequence data and storage for data mining.

3. Kanehisa M. Toward understanding the origin and evolution of cellular organisms. Protein Sci. 28(11), 1947-1951 (2019).

-. Describes the foundation of cellular progressions.

4. Lin T, Lyu P. The application of next generation sequencing in cancer precision diagnosis and target therapy selection. Biomed. J. Sci. Tech. Res. 20(1), 14809-14812 (2019).

5. Salto-Tellez M, Gonzalez de Castro D. Next-generation sequencing: a change of paradigm in molecular diagnostic validation. J. Pathol. 234(1), 5-10 (2014).

-. Describes the practical utility and diagnostic accuracy of next-generation sequencing.

6. Jin L, Zuo XY, Su WY et al. Pathway-based analysis tools for complex diseases: a review. Genom. Proteomics Bioinform. 12(5), 210-220 (2014). 
7. Tyers M, Mann M. From genomics to proteomics. Nature 422(6928), 193-197 (2003).

8. Shoemaker DD, Linsley PS. Recent developments in DNA microarrays. Curr. Opin. Microbiol. 5(3), 334-337 (2002).

9. Mitchell W. Natural products from synthetic biology. Curr. Opin. Chem. Biol. 15(4), 505-515 (2011).

10. Bai X, Zhang W, Orantes L et al. Combining next-generation sequencing strategies for rapid molecular resource development from an invasive aphid species, Aphis glycines. PLoS ONE 5(6), e11370 (2010).

11. Rothberg JM, Leamon JH. The development and impact of 454 sequencing. Nat. Biotechnol. (10), 1117-1124 (2008).

12. Plesa C, Sidore AM, Lubock NB, Zhang D, Kosuri S. Multiplexed gene synthesis in emulsions for exploring protein functional landscapes. Science 359(6373), 343-347 (2018).

13. Harismendy O, Ng PC, Strausberg RL et al. Evaluation of next generation sequencing platforms for population targeted sequencing studies. Genome Biol. 10(3), R32 (2009).

14. Vera JC, Wheat CW, Fescemyer HW et al. Rapid transcriptome characterization for a nonmodel organism using 454 pyrosequencing. Mol. Ecol. 17(7), 1636-1647 (2008).

-. Discusses dynamics in the field of genomics and proteomics alongside current developments in sequencing, synthetic biology and molecular-based assays.

15. Hughes RA, Ellington AD. Synthetic DNA synthesis and assembly: putting the synthetic in synthetic biology. Cold Spring Harb. Perspect. Biol. 9(1), a023812 (2017).

16. Kosuri S, Church GM. Large-scale de novo DNA synthesis: technologies and applications. Nat. Methods 11(5), 499-507 (2014).

17. Mueller S, Coleman JR, Wimmer E. Putting synthesis into biology: a viral view of genetic engineering through de novo gene and genome synthesis. Chem. Biol. 16(3), 337-347 (2009).

18. Roy S, Caruthers M. Synthesis of DNA/RNA and their analogs via phosphoramidite and H-phosphonate chemistries. Molecules 18(11), 14268-14284 (2013).

19. Agarwal KL, Büchi H, Caruthers MH et al. Total synthesis of the gene for an alanine transfer ribonucleic acid from yeast. Nature 227(5253), 27-34 (1970).

20. Khorana HG, Agarwal KL, Besmer P et al. Total synthesis of the structural gene for the precursor of a tyrosine suppressor transfer RNA from Escherichia coli. 1. General introduction. J. Biol. Chem. 251(3), 565-570 (1976).

21. Au LC, Yang FY, Yang WJ, Lo SH, Kao CF. Gene synthesis by a LCR-based approach: high-level production of leptin-L54 using synthetic gene in Escherichia coli. Biochem. Biophys. Res. Commun. 248(1), 200-203 (1998).

22. Xiong AS, Peng RH, Zhuang J et al. Non-polymerase-cycling-assembly-based chemical gene synthesis: strategies, methods, and progress. Biotechnol. Adv. 26(2), 121-134 (2008).

23. Dillon PJ, Rosen CA. A rapid method for the construction of synthetic genes using the polymerase chain reaction. BioTechniques 9(3), 298-300 (1990).

-. Describes various approaches in synthetic DNA.

24. Jayaraman K, Fingar SA, Shah J, Fyles J. Polymerase chain reaction-mediated gene synthesis: synthesis of a gene coding for isozyme c of horseradish peroxidase. Proc. Natl Acad. Sci. USA 88(10), 4084-4088 (1991).

25. Stemmer WP, Crameri A, Ha KD, Brennan TM, Heyneker HL. Single-step assembly of a gene and entire plasmid from large numbers of oligodeoxyribonucleotides. Gene 164(1), 49-54 (1995).

-. Describes the synthesis of a gene and single-step assembly of an entire plasmid from large numbers of oligodeoxyribonucleotides.

26. Horton RM, Hunt HD, Ho SN, Pullen JK, Pease LR. Engineering hybrid genes without the use of restriction enzymes: gene splicing by overlap extension. Gene 77(1), 61-68 (1989).

27. Urban A, Neukirchen S, Jaeger KE. A rapid and efficient method for site-directed mutagenesis using one-step overlap extension PCR. Nucleic Acids Res. 25(11), 2227-2228 (1997).

28. Gao X, Yo P, Keith A, Ragan TJ, Harris TK. Thermodynamically balanced inside-out (TBIO) PCR-based gene synthesis: a novel method of primer design for high-fidelity assembly of longer gene sequences. Nucleic Acids Res. 31(22), e143 (2003)

29. Xiong AS. A simple, rapid, high-fidelity, and cost-effective PCR-based two-step DNA synthesis method for long gene sequences. Nucleic Acids Res. 32(12), e98 (2004).

30. Young L. Two-step total gene synthesis method. Nucleic Acids Res. 32(7), e59 (2004).

31. Hilgarth RS, Lanigan TM. Optimization of overlap extension PCR for efficient transgene construction. MethodsX. 7, 100759 (2020).

32. Kalendar R, Khassenov B, Ramankulov Y, Samuilova O, Ivanov KI. FastPCR: an in silico tool for fast primer and probe design and advanced sequence analysis. Genomics 109(3-4), 312-319 (2017).

33. Camer GA, Oikawa Y, Omaki H, Endoh D. Novel synthetic nucleotides of notifiable dengue (1-4), Japanese encephalitis, yellow fever and Zika flaviviruses. Future Sci. OA 5(1), FSO353 (2018).

34. Kadkhodaei S, Memari HR, Abbasiliasi S et al. Multiple overlap extension PCR (MOE-PCR): an effective technical shortcut to high throughput synthetic biology. RSC Adv. 6(71), 6668266694 (2016).

-. Describes the value of OE-PCR in efficiently generating synthetic nucleotides.

35. Gibson DG, Young L, Chuang RY, Venter JC, Hutchison CA III, Smith HO. Enzymatic assembly of DNA molecules up to several hundred kilobases. Nat. Methods 6(5), 343-345 (2009).

36. Caruthers MH. A brief review of DNA and RNA chemical synthesis. Biochem. Soc. Trans. 39(2), 575-580 (2011).

37. Gibson DG. Oligonucleotide assembly in yeast to produce synthetic DNA fragments. Methods Mol. Biol. 852, 11-21 (2012).

-. Describes the enzymatic assembly of oligonucleotides to sizable polynucleotides. 
